\title{
Flexibility configurations and preventive maintenance impact on job-shop manufacturing systems
}

\author{
Paolo Renna ${ }^{a^{*}}$
}

${ }^{a}$ School of Engineering, University of Basilicata, Via dell'Ateneo Lucano, 1085100 - Potenza, Italy

\begin{tabular}{l}
\hline C H R O N I C L E \\
\hline Article history: \\
Received October 272016 \\
Received in Revised Format \\
December 222016 \\
Accepted February 272017 \\
Available online \\
March 1 2017 \\
\hline Keywords: \\
Machine flexibility \\
Routing flexibility \\
Corrective maintenance \\
Preventive maintenance \\
Simulation
\end{tabular}
A B S T R A C T

\begin{abstract}
Manufacturing systems need to be able to work under the dynamic and uncertain production environment. Machine and routing flexibility combined with preventive maintenance actions can improve the performance of the manufacturing systems under dynamic conditions. This paper evaluates different levels of machine and routing flexibility combined with different degrees of preventive maintenance policy. The performance measures considered are throughput, work in process and throughput. The performance measures are compared with a system without any flexibility and no preventive maintenance actions. Different levels of flexibility and preventive maintenance actions are examined under a simulation environment. The simulation results highlight more important factors for the performance measures and the best combination of the factors to improve the performance.
\end{abstract}

\section{Introduction}

Today's manufacturing environment is characterized by shorter lead time, rapid introduction of new products, smaller lot sizes and higher competitive pressure. In order to stay competitive, the enterprises need to implement a manufacturing system capable of reacting to the market change, rapidly. Firms often increase in product offerings for the competition pressure and this would drive up the variabilities of product demand. Firms can adopt process flexibility to deal with these changes (see Simchi- Levi, 2010 and Cachon and Terwiesch, 2009). A Flexible Manufacturing System (FMS) can be defined as a computer-controlled configuration of semi-dependent workstations and material-handling systems designed to efficiently manufacture various part types with low to medium volumes. It combines high levels of flexibility with high productivity and low level of work-in-process inventory (Jang \& Park, 1996). In particular, the routing flexibility allows to properly respond to a changing environment, so that the possibility of switching products to different machines in the case of environmental changes can reduce the negative impact of variability on the system performance (Das \& Nagendra, 1997). The FMSs with full flexibility face with extra costs of material handling, CNC machines, higher capital investment

* Corresponding author. Tel. : +39 0971205143

E-mail: paolo.renna@unibas.it (P. Renna)

(C) 2017 Growing Science Ltd. All rights reserved. doi: $10.5267 /$ j.ijiec.2017.3.002 
costs, costs of production planning and scheduling for greater complexity of work flow and production capacity. The FMSs are designed to foreseen several production environment conditions in advanced overestimating CNC machines' characteristic, capacity, etc.

Reconfigurable Manufacturing Systems (RMSs) are designed with customized flexibility, which enable combining the advantages of high productivity of dedicated stations with the flexibility of CNCs (ElMaraghy, 2006). RMSs enable the design of manufacturing systems with focused flexibility reducing costs compared to FMSs. Another important method of increasing production line throughput is to improve the availability of machines through preventive maintenance (Patchong \& Willaeys, 2001; Northworthy \& Abdul-Kader, 2004). Preventive maintenance is a proactive approach to increase the reliability of machines over the long term by scheduling planned maintenance actions aimed at the reduction or prevention of sudden breakdowns and failures (Gento \& Redondo, 2003).

The maintenance costs and strategies become more complex with the increase of manufacturing system complexity. Overall maintenance costs in the U.S. were estimated to be over $\$ 600$ billion in 1981 , with the expectation that they would double within 20 years (Heng et al., 2009). Out of these maintenance costs, 33-50\% was estimated to be wasted on ineffective maintenance, which just emphasizes on the need for advanced research in this area (Heng et al., 2009).

The main types of maintenance operations can be classified in Reactive Maintenance (RM) and Preventive Maintenance (PM). RM performs actions when a tool /machine a tool/ machine starts to function improperly or fails completely, while the PM performs actions on a tool/machine before unacceptable behavior occurs. Even though RM is unavoidable, it is usually more costly and time consuming than the PM (Wang, 2002). A proper PM strategy allows to keep the production line on, improves the product quality and avoids losses caused by equipment failures and RM events.

The flexible manufacturing systems consist of machines that can conduct different manufacturing operations with different process times and the degradation of a machine depends highly on the operations performed on that machine. In this context, the maintenance decisions are harder to make. The PM decisions changing the degradation dynamics, but interrupt the production, then the selection of operations executed on a machine affects the maintenance decisions (Zhou et al., 2007a).

This paper studies the benefits of the routing flexibility and preventive maintenance actions under different levels of routing flexibility and the effect of preventive maintenance actions. The level of routing flexibility is evaluated by considering the concept of limited flexibility proposed in the literature in a multi-plant production planning context (Jordan \& Graves, 1995). In particular, products and machines are chained by a minimum number of links and forming the longest close loop. This chain can provide many typical benefits of a totally flexible system (where every machine can process every part, as in a job shop) and of a non-flexible system (where every machine is dedicated to just one part).

The preventive maintenance policy is evaluated by considering the potential impact on the Mean Time Between Failures (MTBF) and on the Mean Time To Repair (MTTR). A simulation environment is developed to test how the level of flexibility and preventive maintenance policy affect the performance of the manufacturing system. The main performance measures are compared to manufacturing systems with dedicated machines (no flexibility) and without preventive maintenance policy. The value added by flexibility and preventive maintenance are evaluated separately and together.

The remaining of the paper is organised as follows. Section 2 discusses the review of the literature, while in Section 3 the manufacturing system context is provided. Section 4 explains the design of the simulation experiments and discusses the numerical results. Finally, in section 5 the conclusions and future development paths are discussed. 


\section{Literature review}

The works proposed in literature focused separately on flexibility issue or preventive maintenance policies. Jordan and Graves (1995) studied flexibility in a multi-plant production planning context. They explained how a chain structure with each source capable of producing only two product types, is a particularly powerful structure that achieves most of the potential benefits from flexibility while keeping costs of the flexibility relatively low. Boyer and Leong (1996) extended the works of Jordan and Graves (1995) to examine the linkage between process and machine flexibility. They show that high machine flexibility contributes to high process flexibility, which increases output figures. Sheikhzadeh et al. (1998) considered the issue of total flexibility versus chaining at the operational level and obtained results similar to Jordan and Graves (1995). They showed that when higher flexibility requires higher capital investment, multiple smaller chains were better options than a single long chain. Baykasoğlu and Durmuşoğlu (2012) analyzed the effects of flexibility on flow time performance of a simulated job shop. For that purpose, several scenarios are developed under four flexibility levels with two different machine selection rule and three types of dispatching rules. Furthermore, the effect of jockeying as a queuing policy on the flow time performance was also investigated through simulation modelling. Results indicated that full flexibility was a preferable state for most of the cases. However, in some cases, chain configurations perform similar results since it combines the benefits of pooling and specialization. Joseph and Sridharan $(2011,2012)$ developed a simulation analysis to investigate three routing flexibility levels, five sequencing flexibility levels, and four scheduling rules for part sequencing decision are considered for detailed investigation. The system work load characterized by the mean inter-arrival time of parts has been set at different levels. The performance of the FMS was evaluated using various measures related to flow time and tardiness of parts.

Iravani et al. (2011) focused on the problem of ranking (according to average wait in queue) alternative system designs that vary by capacity and the structure of capabilities for open, parallel queueing networks with partially flexible servers. This article developed the Capability Flexibility (CF) index methodology to extend the range of operational environments and designed which could be ranked (as a relatively tractable yet powerful extension of the SF method introduced in Iravani et al., 2005). The CF methodology not only offers insight into how the interaction of capacity and flexibility can improve performance but also offers a fast numerical algorithm for decision support in place of system simulation. For example, our methodology assists in the decision of whom to cross-train for what tasks in service centers with a budget/limit on the amount of multi functionality by creating designs yielding robust performance despite demand uncertainty.

Andradóttir et al. (2013) provided means to identify flexibility structures that are throughput optimal and adaptable to changes in the environment, manifested by perturbations in arrival and/or service rates. To accomplish this, they introduced the notion of a bottleneck set of tasks to queueing networks with flexible, heterogeneous servers, so that the bottleneck set may include several queues and servers. As a result, they identified minimal conditions that should be required of any flexibility structure, if possible.

Shi et al. (2015) developed a theory for the design of process flexibility in a multi-period production system. They proposed and formalized a notion of "effective chaining" termed the Generalized Chaining Condition (GCC), which includes the chaining structure put forth by Jordan and Graves (1995) as a special case. The goal of this paper was to make progress towards the better understanding of the key design principles of process flexibility structures in a multi-period environment, the study of which has been limited due to its inherent complexity.

Wang and Zhang (2015) analyzed the long chain in a distributional robust setting when only the first two moments of the demand are known. Tanrisever et al. (2012) studied chaining and partial flexibility structures under a multi-period MTO environment. They applied a sampling-based decomposition method to devise a feasible production scheduling policy, and used the policy to evaluate the 
effectiveness of different flexibility structures in simulations. Muriel et al. (2006) studied the impact of increasing flexibility at the operational level, and found that limited flexibility could increase production variability more than total flexibility. Bose et al. (2016) addressed the strategic capacity planning problem in a multi-product, multi-plant setting under demand uncertainty. In this paper, they developed a twostage stochastic programming model to determine the capacity and the product-plant configuration to maximize the expected profit.

Renna (2012) studied the manufacturing system performance in dynamic conditions when different maintenance policies are implemented in a multi-machine manufacturing system controlled by multiagent-architecture. There are two extreme maintenance policies that can be applied: no preventive maintenance, where action is taken on the failure state, and intensive preventive maintenance, which can eliminate unforeseen failures, but at a high cost. Dynamic policy maintenance was proposed to reduce the number of maintenance operations of the preventive policy.

Zhou et al. (2007) developed an integrated reconfiguration and age-based maintenance policy on a singleproduct parallel-serial system with reconfiguration capabilities. Reconfigurations consisted of transferring flexible operations to a different machine from the one to which they were initially assigned to, if that move was deemed to be more cost effective. It has been shown in their study that for a wide spectrum of parameters, the policy outperforms the traditional age-based maintenance policy in terms of lower expected total cost, smaller number and amount of unscheduled downtime and higher productivity. Zhou et al. (2007b) used a simulation-based optimization in a load-sharing system, where different components of the system share an overall load. In such systems, loads allocated to each machine vary, which affects degradation dynamics of each machine (higher loads leading to faster degradation and vice versa). It was shown that integrated load allocation and condition-based maintenance policy introduced in that paper results in the increased availability of equipment and more cost-effective operations of the system, when compared to the traditional policies.

Celen and Djurdjanovic (2015) devised an integrated decision-making policy for maintenance scheduling and production sequencing, with the objective of optimizing a customizable objective function, while taking into account operation-dependent degradation models and a production target. Optimization was achieved using a metaheuristic method based on the results of discrete-event simulations of the target manufacturing system. The results show that jointly making maintenance and production sequencing decisions consistently and often significantly outperforms the current practice of making these decisions separately.

Nahas (2017) studied the optimal preventive maintenance policy and the optimal buffer allocation, which minimizes the total system cost subject to a given system throughput level. They assumed that the mean time between failures of all machines will be increased after performing periodic preventive maintenance. An analytical decomposition-type approximation was used to estimate the production line throughput. The optimal design problem was formulated as a combinatorial optimization one where the decision variables are buffer levels and times between preventive maintenance.

The main limits of the literature analyzed can be summarized as follow, and the research proposed outcomes the above limits in the following issues:

- The works proposed in literature does not evaluate the effect of preventive maintenance considering the level of machine flexibility of the manufacturing systems;

- The evaluation of the combined effect of machine flexibility and improvements due to the preventive maintenance policy on the availability of the manufacturing system;

- The development of simulation environment allows to evaluate the above issues in generalized conditions of inter-arrival parameter and routing of the parts. 


\section{Reference context}

The manufacturing system consists of three machines capable of manufacturing the operations required by the parts. Three configurations are considered: no flexibility, limited flexibility and full flexibility. Each machine $\left(w s_{i}\right)$ is able to perform one manufacturing operation in the dedicated configuration (Fig. 1a). In case of limited flexibility, each machine is able to perform two manufacturing operations (Fig. 1b). Finally, each machine is able to perform all manufacturing operations in the full flexibility case (Fig. 1c).

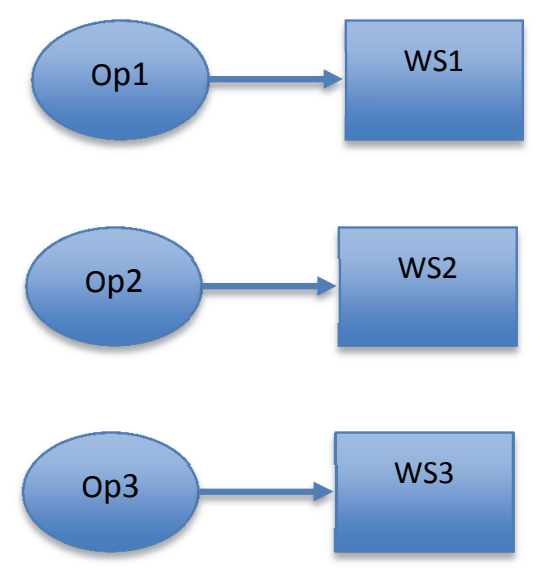

Fig. 1a. Dedicated

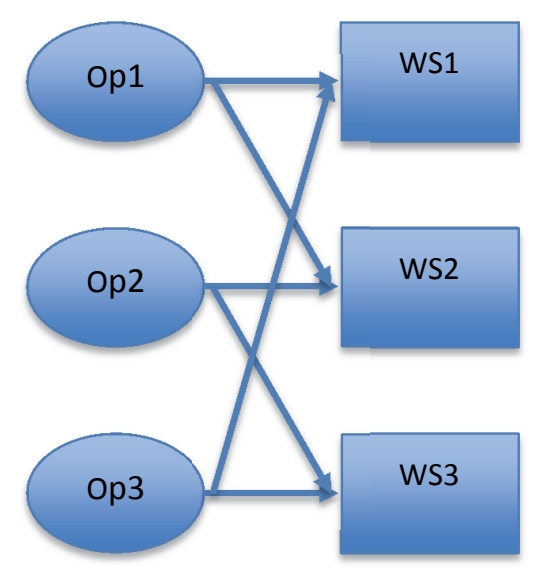

Fig. 1b. Limited flexibility

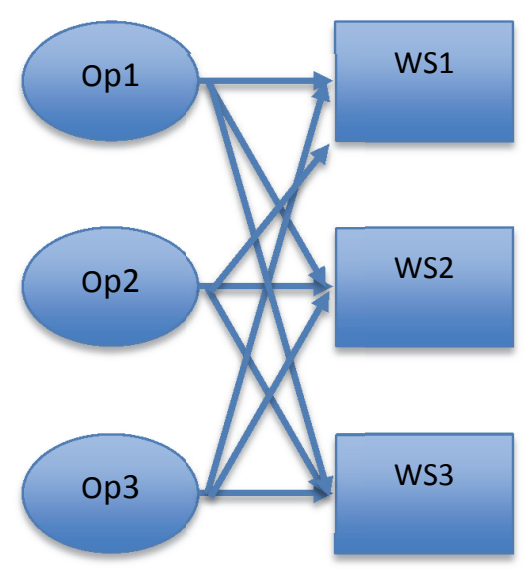

Fig. 1c. Full flexibility

Different configurations with the increasing of the manufacturing operations are assigned to the machines, which yields different costs. The increasing of manufacturing operations assigned to a machine is performance with additional investment (e.g., flexible material handling, programmable machines, and flexible workforce) or higher operating costs (e.g., more frequent setups, increased material handling, increased control complexity), therefore increased resource sharing could result in a trade-off between performance and cost. Each machine is affected by failures; the Mean Time Between Failure and the Mean Time to Repair a machine are supposed to be exponentially distributed with the mean time between failure (MTBF) and mean time to repair (MTTR), respectively.

A Preventive Maintenance (PM) policy is performed on each machine; the actions are performed on each Machine $M_{i}$ every period $t_{i}$. It is assumed that as the preventive maintenance frequency increases, the MTBF will generally increase (Meller \& Kim, 1996). The following parameters are defined:

1. Min: present MTBF with no PM program.

2. Max: maximum MTBF possible with a very frequent PM program.

3. $\beta$ : Shape factor for the asymptotic gain in PM.

There are many possible ways in which to model the relationship of these parameters to the potential impact of the PM program (Meller \& Kim, 1996). In this paper, the form presented by Groenevelt et al. (1992) and Meller and Kim (1996) is adopted:

$$
M T B F=\min +(\max -\min ) \times\left(1-e^{\beta / t i}\right) .
$$

The shape factor for the asymptotic gain in PM $(\beta)$ can be also obtained using the following equation (Meller \& Kim, 1996):

$$
\beta=-R \ln (1-(\alpha \times \max -\min ) /(\max -\min ))
$$


The part enters in the manufacturing systems following an exponential distribution. When a part enters the process plan is assigned in terms of:

- Number of operations to perform extracted by a uniform distribution between 1 and 3;

- The process time assigned to each operation extracted by a uniform distribution between 5 and 10 unit-time.

The load of the resources in case of limited and full flexibility is performed as follows.

$$
W L_{j}(t)=\sum_{i=1}^{N Q_{j}} t p_{i}+\operatorname{trem}_{j}(t) \times X_{j}+\operatorname{EXP}(M T T R)_{j} \times F_{j}
$$

where,

$t p_{i}$ is the process time of the part $\mathrm{i}$ in queue;

trem $_{j}$ is the remaining process time of the part in process in machine $\mathrm{j}$ at time $\mathrm{t}$ if the machine is busy ( $X_{j}=1$ if the machine is busy, $X_{j}=0$ otherwise).

$\operatorname{EXP}(M T T R)$ is the expected MTTR if the machine is in state of failure $\left(F_{j}=1\right.$ if the machine is in failure state, $F_{j}=0$ otherwise).

When the part enters at time $t$, it is loaded to the machine with lower $W L_{j}(t)$ compatible with the manufacturing operation required by the part. Following this strategy, there is not sequence constraints among the manufacturing operations assigned to the process plan of the parts.

\section{Simulation environment}

A simulation environment has been developed to evaluate the performance measures of the maintenance policy in a manufacturing system with dedicated, limited and full flexibility. The author selected the Simul8 ${ }^{\circledR}$ discrete event simulation platform for the development of the simulation models. Six models are developed as shown in Fig. 2; for each flexibility level is modeled the case with and without preventive maintenance.
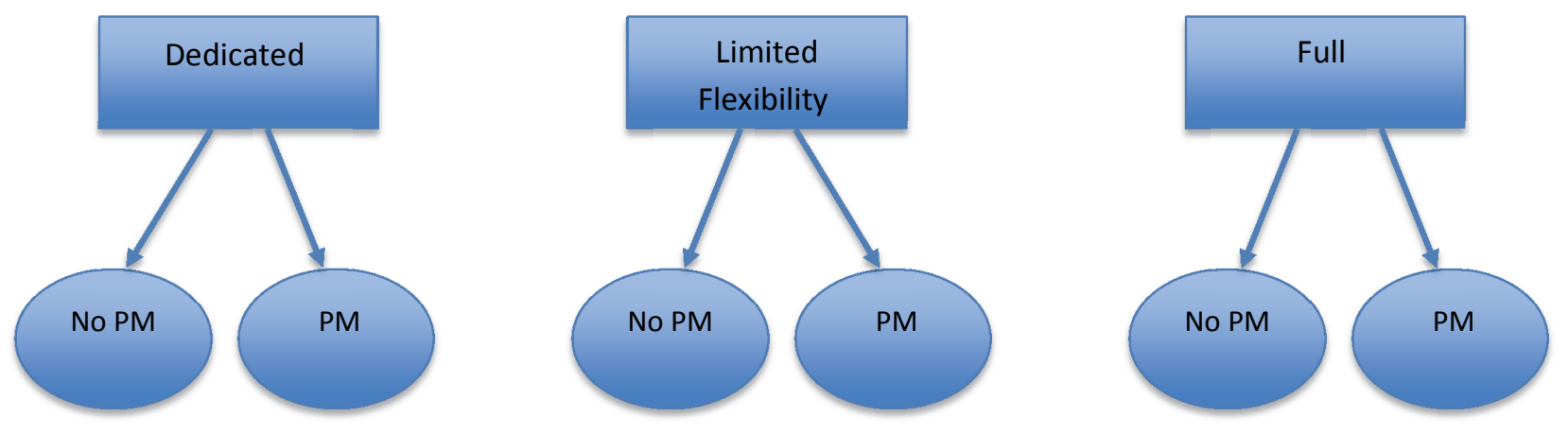

Fig. 2. Simulation models

The data of the simulation models are reported in Table 1. MTBF follows an exponential distribution with a minimum value of 500 (corrective maintenance without any preventive actions) and a maximum value of 5000. It is considered a periodic time between PM actions of 500 unit-time.

The simulations have been conducted for different values of $\beta$ (see Eq.(2)); this value determines how the PM actions affect the MTBF. A greater value of $\beta$ leads to a greater MTBF. In case of corrective maintenance policy, the MTTR follows an exponential distribution with a parameter of 100 . When the PM actions are performed, it is considered a reduction of the MTTR parameter; in particular, there are considered the following reduction: $10 \%, 20 \%, 30 \%$ and $40 \%$. Finally, three levels of the inter-arrival parameter are considered to evaluate different degree of manufacturing utilization. 
Table 1

Simulation data

\begin{tabular}{|c|c|c|c|c|}
\hline & Min & Max & & \\
\hline MTBF EXPO & 500 (MTBFc) & 5000 & & \\
\hline $\mathrm{t}_{\mathrm{i}}$ [actions/time] & 500 & & & \\
\hline beta & $1-7-14-21-29$ & & & \\
\hline MTTRc EXPO & 100 & & & \\
\hline MTTRp [k] & 0.9 & 0.8 & 0.7 & 0.6 \\
\hline Models & No flex & Flex 2 & full & \\
\hline Expo inter-arrival & 6 & 5 & 4 & \\
\hline Number of operations & UNIF[1,3] & & & \\
\hline Processing time & UNIF $[5,10]$ & & & \\
\hline
\end{tabular}

The complete simulation experiments concern $5 \beta \times 4 \mathrm{k} \times 3$ inter-arrival $\times 3$ models (with PM policy) + 3 inter-arrival $\times 3$ models $=189$ classes simulated (Table 1$)$. For each experimental class, the number of replications enables us to ensure a 5\% confidence interval and 95\% confidence level for each performance measure. The performance measures investigated are the following.

- Average throughput time of the parts. This is the average time that the typology parts spend in the manufacturing system;

- Throughput of the manufacturing system;

- Work in process (WIP) of the manufacturing system.

- Average utilization of the manufacturing system;

- Total number of corrective maintenance operations;

The numerical results are reported as a percentage difference compared to the benchmark case (pure corrective maintenance policy). Fig. 3 shows the percentage difference of the work in process and throughput time (two performance measures have the same behavior) for each model compared to the case without preventive maintenance for the inter-arrival 6 . The abscissa reports the $\mathrm{MTTR}_{\mathrm{p}}$ parameter.
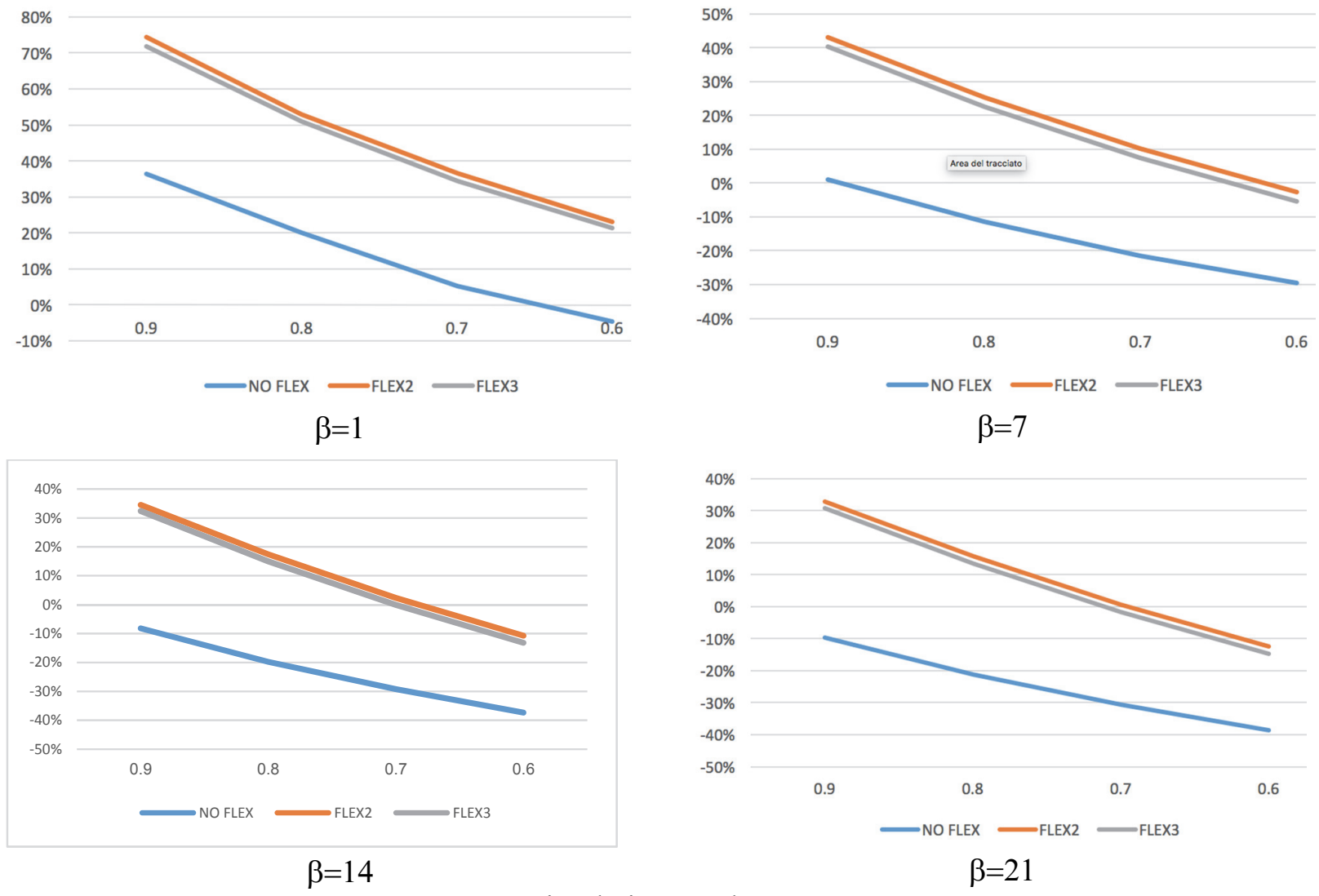

Fig. 3. Simulation results - EXPO 6 
The model without flexibility has greater improvements by the preventive maintenance policy. The limited and full flexibility are very similar. Moreover, the values of $\beta$ 7,14 and 21 lead to the same results. The value of $\beta=1$ leads to very low improvements only for the case of no flexibility of the manufacturing system. When $\beta=7$, no flexibility configuration has improvements when the MTTR is reduced from $20 \%$. The limited and full flexibility have improvements from $\beta=7$ and the MTTR reduces of $40 \%$ due to the preventive maintenance policy. These results highlight the necessary effect in terms of increment of MTBF ( $\beta$ parameter) and MTTR reduction ( $\mathrm{k}$ parameter) to improve the performance of each configuration under preventive maintenance policy.

Fig. 4 shows the same results when the inter-arrival parameter is 5 . The results are similar for $\beta=1,7$ and 14 as the above results discussed. The improvements are closer than the case with higher inter-arrival. Generally, the improvements are higher than the above case and with lower reduction of MTTR. Therefore, the higher manufacturing utilization leads to reach similar results among the flexibility configurations and higher improvements.
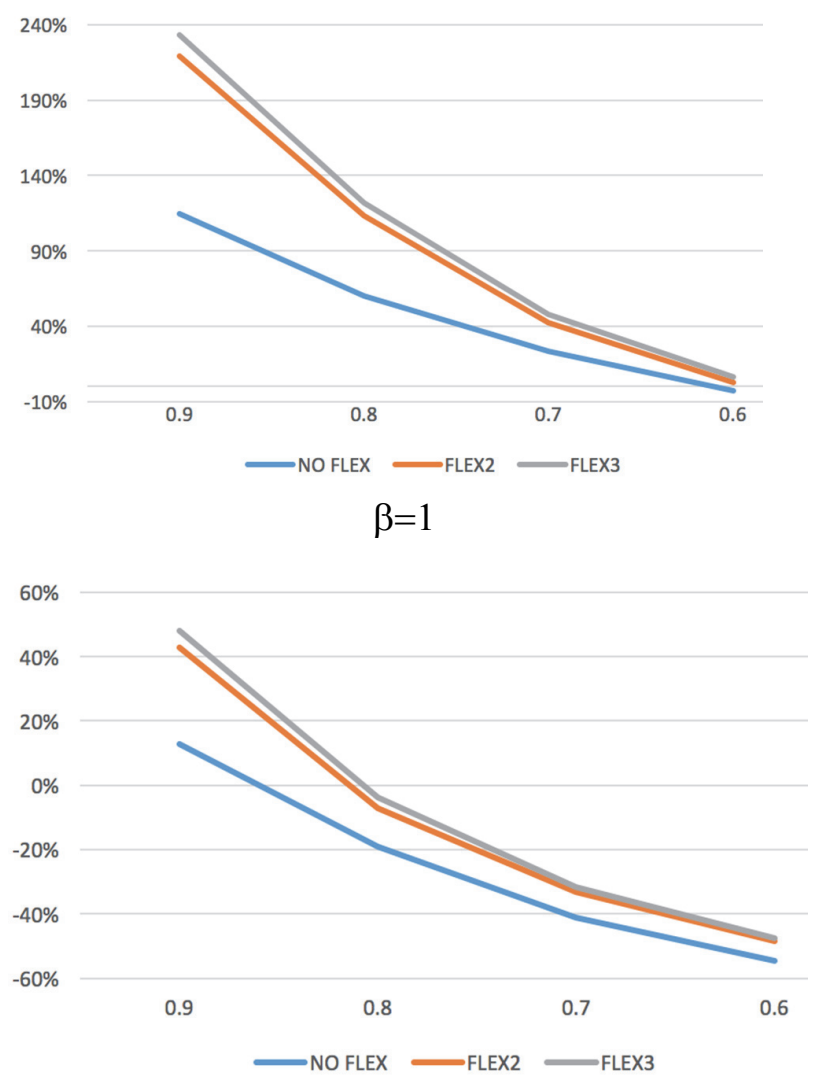

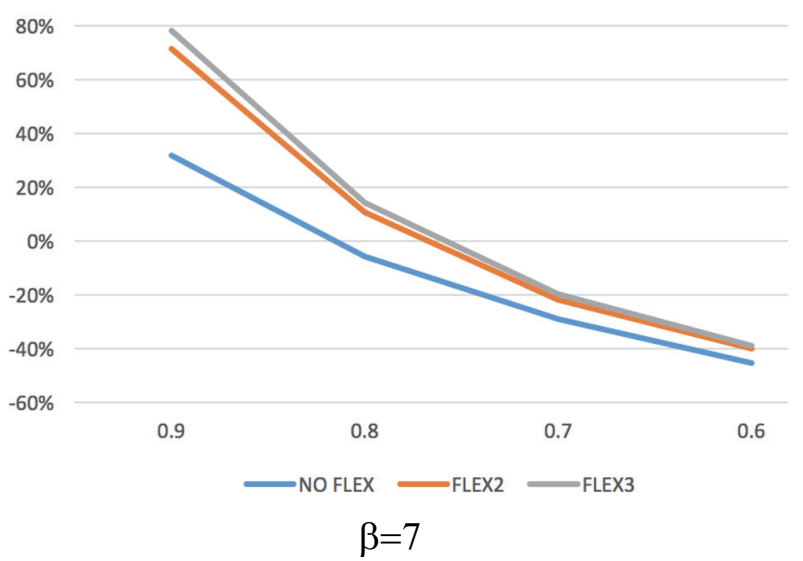

Same results for other $\beta$ values.

$$
\beta=14
$$

Fig. 4. Simulation results EXPO 5

Fig. 5 shows the simulation results for the inter-arrival parameter 4 . In this case, the flexibility configurations have the same results. Therefore, when the manufacturing utilization is very high the preventive maintenance policy leads to the same improvements independently by the flexibility configurations. The cases of $\beta 7$ and 14 lead to relevant improvements from MTTR reduction of $30 \%$. 

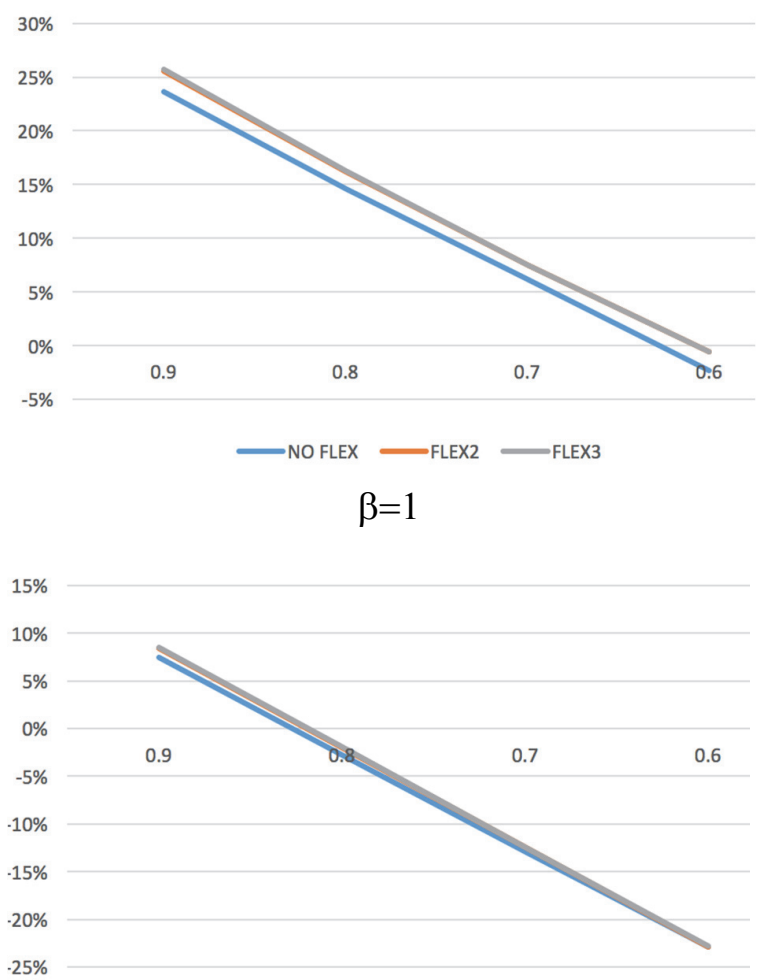

$\cdot 25 \%$

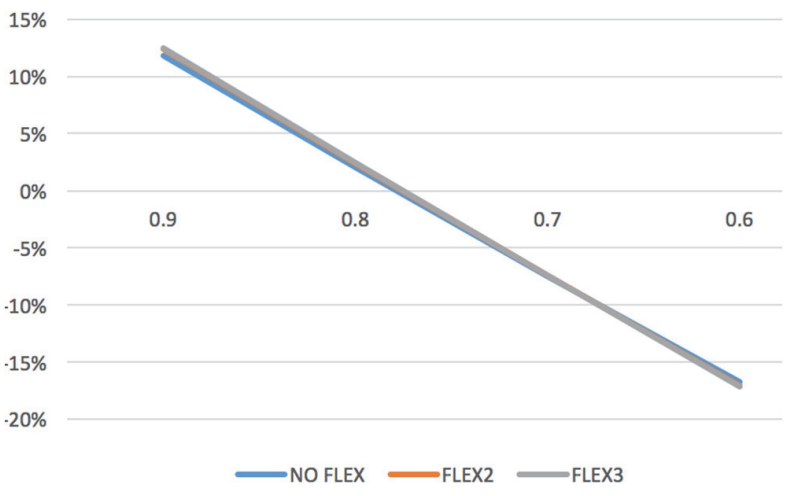

$\beta=7$

Same results for other $\beta$ values.

$$
\beta=14
$$

Fig. 5. Simulation results EXPO 4

Table 2 reports the average WIP for each parameter evaluated. The average manufacturing utilization leads to greater improvements when is higher (EXPO 4). The configuration with higher improvements due to the preventive maintenance is the no flexibility. The parameter $k$ (MTTR reduction) has the higher importance to obtain the better performance.

Table 2

Simulation results WIP

\begin{tabular}{ccccc}
\hline & \multicolumn{3}{c}{ AVERAGE COMPUTATION } & \\
\hline EXPO 6 & $7.28 \%$ & NO FLEX & $-9.08 \%$ \\
EXPO 5 & $8.06 \%$ & & FLEX 2 & $9.26 \%$ \\
EXPO 4 & $-2.52 \%$ & FLEX 3 & $9.95 \%$ \\
\hline beta 1 & beta 7 & beta 14 & beta 21 & beta 29 \\
$42.89 \%$ & $28.41 \%$ & $17.20 \%$ & $8.64 \%$ & $1.53 \%$ \\
\hline $\mathrm{K}=0.9$ & $\mathrm{~K}=0.8$ & $\mathrm{~K}=0.7$ & $\mathrm{~K}=0.6$ & \\
\hline $36.51 \%$ & $9.51 \%$ & $-9.46 \%$ & $-23.06 \%$ & \\
\hline
\end{tabular}

The results are very similar for the throughput time of the parts (Table 3).

Table 3

Simulation results - throughput time

\begin{tabular}{ccccc}
\hline & & AVERAGE COMPUTATION & \\
\hline EXPO 6 & $7.42 \%$ & & NO FLEX & $-9.04 \%$ \\
EXPO 5 & $8.07 \%$ & & FLEX 2 & $9.45 \%$ \\
EXPO 4 & $-2.38 \%$ & FLEX 3 & $10.05 \%$ \\
beta 1 & beta 7 & beta 14 & beta 21 & beta 29 \\
$42.63 \%$ & $28.30 \%$ & $17.19 \%$ & $8.72 \%$ & $1.68 \%$ \\
K=0.9 & $\mathrm{K}=0.8$ & $\mathrm{~K}=0.7$ & $\mathrm{~K}=0.6$ & \\
$36.40 \%$ & $9.59 \%$ & $-9.26 \%$ & $-22.79 \%$ & \\
\hline
\end{tabular}


The throughput is the performance with lower difference (see Table 4).

Table 4

Simulation results - throughput

\begin{tabular}{ccccc}
\hline & \multicolumn{3}{c}{ AVERAGE COMPUTATION } & \\
\hline EXPO 6 & $0.11 \%$ & & NO FLEX & $0.49 \%$ \\
EXPO 5 & $-0.11 \%$ & & FLEX 2 & $0.21 \%$ \\
EXPO 4 & $0.75 \%$ & FLEX 3 & $0.21 \%$ \\
beta 1 & beta 7 & beta 14 & beta 21 & beta 29 \\
$-1.23 \%$ & $-0.77 \%$ & $-0.35 \%$ & $0.03 \%$ & $0.41 \%$ \\
K=0.9 & $\mathrm{K}=0.8$ & $\mathrm{~K}=0.7$ & $\mathrm{~K}=0.6$ & \\
$-1.23 \%$ & $-0.08 \%$ & $0.83 \%$ & $1.77 \%$ & \\
\hline
\end{tabular}

Tables 2, 3 and 4 summarize the parameters with higher importance and the performance with higher improvements. Fig. 6 shows the number of corrections activities by considering the values of inter-arrival and beta. The values are very similar for the configurations tested. The inter-arrival parameter is relevant only for the case of pure corrective maintenance policy; when the preventive maintenance is performed the results is the same for the three inter-arrival parameters. The value of beta 14 is enough to obtain a null number of correction actions; which is relevant when the correction action can affect the quality of the product in machine.

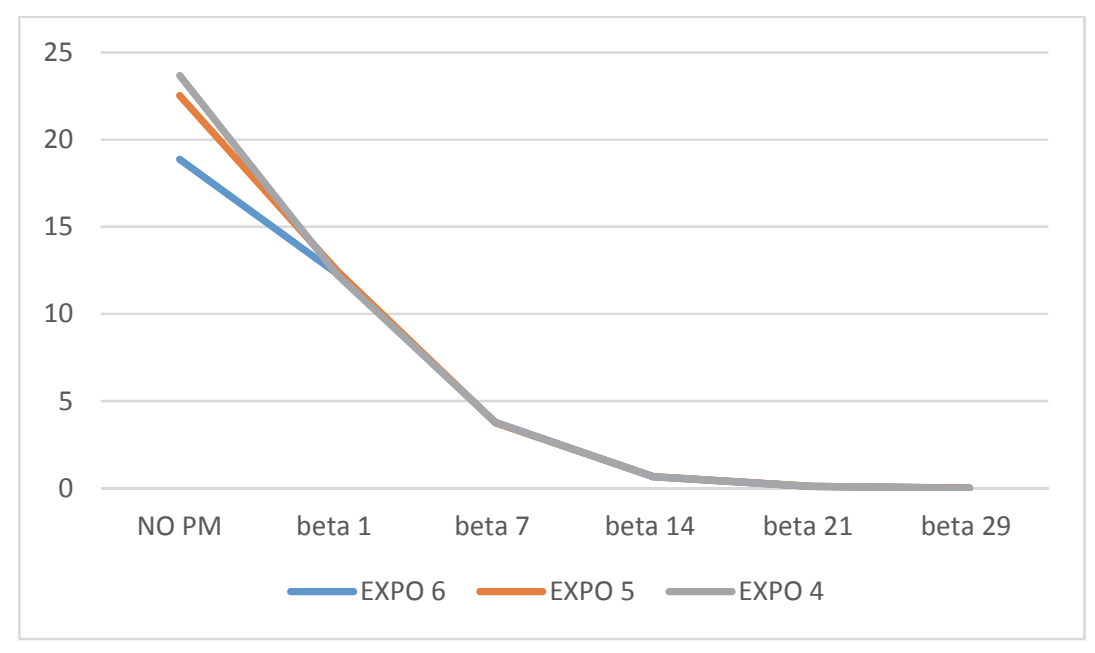

Fig. 6. Number of corrections activities

\section{Conclusions and future development}

The research presented in this paper is concerned with the evaluation of the flexibility configurations of a manufacturing system with the introduction of a preventive maintenance policy. The simulation environment developed has been used to evaluate the impact of the preventive maintenance policy on the flexibility configurations when the manufacturing utilization changes and with different parameters of the maintenance policy. The utilization of the manufacturing system is relevant to the impact of the preventive maintenance; in case of low utilization, the configuration without flexibility has the greater improvements by the introduction of the preventive maintenance. When the utilization of the manufacturing system increases the benefits of the preventive maintenance are the same for all configurations.

The evaluation of the parameters tested highlights how the utilization and the reduction of MTTR have the main effect on the improvement of the performance measures. This means that the preventive maintenance policy should improve also the MTTR to become a real value added for the manufacturing 
system. The simulation environment developed can be a valid support decision system to evaluate the parameters of the preventive maintenance policy in terms of reduction of MTTR and increment of MTBF necessary to improve the manufacturing system performance.

Future development paths should investigate manufacturing systems more complex as the cellular manufacturing system. Moreover, the evaluation of the products quality due to the preventive maintenance policy can be studied.

\section{References}

Andradóttir, S., Ayhan, H., \& Down, D. G. (2013). Design principles for flexible systems. Production and Operations Management, 22(5), 1144-1156.

Baykasoğlu, A., \& Durmuşoğlu, Z. D. (2012). Flow time analyses of a simulated flexible job shop by considering jockeying. The International Journal of Advanced Manufacturing Technology, 58(5-8), 693-707.

Bose, D., Chatterjee, A. K., \& Barman, S. (2016). Towards dominant flexibility configurations in strategic capacity planning under demand uncertainty. OPSEARCH, 53(3), 604-619.

Boyer, K. K., \& Leong, G. K. (1996). Manufacturing flexibility at the plant level. Omega, 24(5), 495510.

Cachon, G., \& Terwiesch, C. (2009). Matching supply with demand. $2^{\text {nd }}$ ed. McGraw-Hill.

Celen, M., \& Djurdjanovic, D. (2015). Integrated Maintenance Decision-Making and Product Sequencing in Flexible Manufacturing Systems. Journal of Manufacturing Science and Engineering, 137(4), 041006.

Das, S. K., \& Nagendra, P. (1997). Selection of routes in a flexible manufacturing facility. International Journal of Production Economics, 48(3), 237-247.

Gento, A. M., \& Redondo, A. (2003). Rough sets and maintenance in a production line. Expert Systems with Applications, 20(5), 271-279.

Groenevelt, H., Pintelon, L., \& Seidmann, A. (1992). Production batching with machine breakdowns and safety stocks. Operations Research, 40(5), 959-971.

Heng, A., Zhang, S., Tan, A. C., \& Mathew, J. (2009). Rotating machinery prognostics: State of the art, challenges and opportunities. Mechanical systems and signal processing, 23(3), 724-739.

Iravani, S.M., Kolfal B., \& Van Oyen, M.P. (2011). Capability flexibility: a decision support methodology for parallel service and manufacturing systems with flexible servers. IIE Transactions, 43(5), 363-382

Iravani, S.M.R., Van Oyen, M.P., \& Sims, K.T. (2005). Structural flexibility: a new perspective on the design of manufacturing and service operations. Management Science, 51(2), 151-166.

Jang, S. Y., Park, J., \& Park, N. (1996). An integrated decision support system for FMS production planning and scheduling problems. International Journal Advance Manufacturing Technology, 11, $101-110$.

Jordan, W.C., \& Graves, S.C. (1995). Principles on the benefits of manufacturing process flexibility. Management Science, 41(4), 577- 594.

Joseph, O.A., \& Sridharan, R. (2011). Effects of routing flexibility, sequencing flexibility and scheduling decision rules on the performance of a flexible manufacturing system. The International Journal of Advanced Manufacturing Technology, 56, 291-306.

Joseph, O.A., \& Sridharan, R. (2012) Effects of flexibility and scheduling decisions on the performance of an FMS: simulation modelling and analysis, International Journal of Production Research, 50(7), 2058-2078.

ElMaraghy, H. A. (2005). Flexible and reconfigurable manufacturing systems paradigms. International Journal of Flexible Manufacturing Systems, 17(4), 261-276.

Meller, R. D., \& Kim, D. S. (1996). The impact of preventive maintenance on system cost and buffer size. European Journal of Operational Research, 95(3), 577-591. 
Muriel, A., Somasundaram, A., \& Zhang, Y. (2006). Impact of partial manufacturing flexibility on production variability. Manufacturing \& Service Operations Management, 8(2), 192-205.

Nahas, N. (2017). Buffer allocation and preventive maintenance optimization in unreliable production lines. Journal of Intelligent Manufacturing, 28(1), 85-93.

Northworthy, S., \& Abdul-Kader, W. (2004). Impact of preventive maintenance on serial production line performance: a simulation approach. In ASAC 2004, Quebec City, Quebec.

Patchong, A., \& Willaeys, D. (2001). Modeling and analysis of an unreliable flow, line composed of parallel-machine stages. IIE Transactions, 33, 559-568.

Renna, P. (2012). Influence of maintenance policies on multi-stage manufacturing systems in dynamic conditions. International Journal of Production Research, 50(2), 345-357.

Sheikhzadeh, M., Benjaafar, S., \& Gupta, D. (1998). Machine sharing in manufacturing systems: Total flexibility versus chaining. International Journal Flexible Manufacturing Systems, 10(4), 351-378.

Shi, Cong and Wei, Yehua and Zhong, Yuan, Process Flexibility for Multi-Period Production Systems (September 3, 2015). Available at SSRN: http://ssrn.com/abstract $=2655790$ or http://dx.doi.org/10.2139/ssrn.2655790

Simchi-Levi, D. (2010). Operations Rules: Delivering Customer Value through Flexible Operations. MIT Press.

Tanrisever, F., D. Morrice, D. Morton. (2012). Managing capacity flexibility in make-to-order production environments. European Journal of Operational Research, 216(2) 334-345.

Wang, X., \& Zhang, J. (2015). Process flexibility: A distribution-free bound on the performance of kchain. Operations Research, 63(3), 555-571.

Wang, H. (2002). A Survey of Maintenance Policies of Deteriorating Systems. European Journal of Operational Research, 139(3), 469-489.

Zhou, J., Djurdjanovic, D., Ivy, J., \& Ni, J. (2007a). Integrated Reconfiguration and Age-Based Preventive Maintenance Decision Making. IIE Transactions, 39(12), 1085-1102.

Zhou, J., Djurdjanovic, D., Ivy, J., \& Ni, J. (2007b). Integrated Load-Allocation and Condition-Based Maintenance Policy in a Multi-Unit Load-Sharing Deteriorating System. Proceedings of the 61st Meeting of the Society for Machinery Failure Prevention Technology, Virginia Beach, VA, pp. 215228.

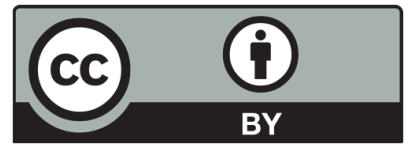

(C) 2017 by the authors; licensee Growing Science, Canada. This is an open access article distributed under the terms and conditions of the Creative Commons Attribution (CCBY) license (http://creativecommons.org/licenses/by/4.0/). 